\title{
U-Mentalism Patent: THE BeginNINg OF Cinematic SuPERCOMPUTATION
}

\author{
Luís Homem
}

Centro de Filosofia das Ciências da Universidade de Lisboa

\begin{abstract}
This paper discloses in synthesis a super-computation computer architecture (CA) model, presently a provisional Patent Application at INPI ( $n^{\circ}$ 116408). The outline is focused on a method to perform computation at or near the speed of light, resorting to an inversion of the Princeton CA. It expands from isomorphic binary/RGB (typical) digital "images", in a network of (UTM)s over Turing-machines (M)s. From the binary/RGB code, an arithmetic theory of (typical) digital images permits fully synchronous/orthogonal calculus in parallelism, wherefrom an exponential surplus is achieved. One such architecture depends on any "cell"-like exponential-prone basis such as the "pixel", or rather the RGB "octet-byte", limited as it may be, once it is congruent with any wave-particle duality principle in observable objects under the electromagnetic spectrum and reprogrammable designed. Well-ordered instructions in binary/RGB modules are, further, programming composed to alter the structure of the Internet, in virtual/virtuous eternal recursion/recurrence, under man-machine/machine-machine communication ontology.
\end{abstract}

\section{KEYWORDS}

U-Mentalism, Super-computation, Computer Architecture, Cybernetics, Programming Languages Design

\section{INTRODUCTION}

This document is intended to serve as white paper to describe in the most possible composed details and in anticipation the technology of U-Mentalism. As referred beforehand "U-Mentalism is a philosophical and programming idea that proposes a singular (one only and individual, intensional) and universal (all and wholly comprehensive, extensional) programming language which is, simultaneously, an inverted scheme of all the established computer architectures (...)"[1], with this meaning a common ever-evolving Assembly Programming Language, giving rise to a semantic explosion of programming languages, all throughout what can be described as an inversion of the so called von Neumann or Princeton CA in network cybernetic fashion. Protected as it may be by a provisional Patent Application at INPI (Portuguese Institute of Industrial Property) ( $\mathrm{n}^{\mathrm{o}}$ 116408), a fairly elaborated disclosure can be eloquent enough as to describe its most basic settings. Although fundamentally expanded in technical computational terms, it should always be attained that one such implementational, informatic and informational method [U-Mentalism and the "C" approach in computation] is inextricable from a metaphysical naturalistic method [U-Mentalism and the "O" approach in ontology]. In addition to this, it is also to remember that the shades of relativistic and possibly technical contentious matter are all related to the perdurable problem of the context and contingent matter of technology's state-ofthe-art, yet never to the very core of the new utility general-purpose application or, better said, the invention's original idea. Lastly, it is worth mentioning that the technical drawing of the CA herein disposed can also be found in the following divulging website: www.u-mentalism.com . 
International Journal of Web \& Semantic Technology (IJWesT) Vol.12, No.1, January 2021

\subsection{The Imagetic Frame of Reference of U-Mentalism in Relation to the "O(Ntological)" and "C(Omputational)" Approaches}

We shall begin by stating that the "C" method is to be performed by UTM(s) with controlled, orthogonal and synchronous, camera-like digital images RGB sensing/processing and computing binary isomorphic processors, thus with (typical) digital images "scanner" (impression) and "printer" (emission) abilities. Universal Turing Machines are described in .6 "The universal computing machine" of Alan Turing's 1936 seminal paper On Computable Numbers, with an Application to the Entscheidungsproblem [2]. In this paragraph the machine $\mathrm{U}$ is supplied with a tape where is ahead written the S. D. (standard description) for any other machine M. In such manner, $\mathrm{U}$ will compute exactly the same sequence as the machine $\mathrm{M}$. The novelty herein in $\mathrm{U}$ Mentalism is that the U-machine or $\operatorname{UTM}(\mathrm{s})$ are camera image sensing (impression) and processing (emission) RGB/binary isomorphic processing computers with likewise symbols and $\mathrm{m}$-configurations, and because $\mathrm{UTM}(\mathrm{s})$ are also $\mathrm{M}(\mathrm{s})$, every UTM is also able to compute any other UTM(s)' computable sequence, and in such a way that any such computable sequence in a network of information, or the Internet, is equally likely to be computed. In UTM(s) the graphical camera display is the forefront processor, and RGB, although synchronous to the binary code, is the primeval symbolic feedback, in exact opposition to classical computation in $M(s)$, wherein control and communication is wholly set on the binary code.

Now, in U-Mentalism under the "O" approach, what is relevant is the constitution of the physicalist most differentiated quanta of spacetime and observables, each of which to bear all possible viewpoint "images" for every possible and most differentiated quanta of spacetime and observables, wherein the latter observables are themselves included, as well as every all other, thus conceivably measured, in every possible viewpoint "images" in spacetime, infinitely and recursively. All in all, one such cogitation is produced by a pure imagetic frame of reference of spacetime with bijective transformations of the state spacetime common to the different observers, and wherein the proper metric/imagetic/recursive and observable state spacetime is the observational reference frame of spacetime itself. All in all, in an analogy argument it goes as if spacetime, not affected by the indeterminacy principle (Heisenberg) and in full entanglement, could infinitely observe itself with the frame of reference being any constituted chosen metric and noematic image. If, for example, we could, non-contradictorily, in inter-noumenal or intermonadic fashion, observe every viewpoint of every photon for the most differentiated quanta of spacetime observables, recursively in a standard description (S.D.) frame of reference of imagetic nature, herein presumed the pure imagetic viewpoint of photons, we are more inclined to understand not only the observables incompleteness (roughly expanding Gödel's theorems from logic to "O") and undefinability (roughly expanding Tarski's theorem from logic to "O"), but also, more conveniently, the consistent and effective passible intermediate states if the set of boundaries or limits are much less forceful. One such case is, most definitely, the "(typical) digital image", a conveniently neutral physicalist viewpoint, further permitting the classes of computable expressions and functions, such as the binary code at its core, to be mapped onto images, as expressions of the RGB codomain. We choose the S.D. frame of reference to be, according to the state-of-the-art technology, the $8 \mathrm{~K}(\approx 8000$ Pixels) 60 Frames per Second (FPS), 24 bits in depth images, choosing pixels-per-inch (PPI) as the standard resolution pattern, in which case we are exhibiting a basic setting for U-Mentalism under the "C" approach. In this wise, although far away from the philosophical crux of U-Mentalism "O" - "every possible image in every possible spacetime composed in every possible mind and n-dimensionally by perceived photons of light" [1] -we are resolutely bridging the chiasm by means of the presentation of a simple object, i.e., an $\mathrm{U}$. 
International Journal of Web \& Semantic Technology (IJWesT) Vol.12, No.1, January 2021

\subsection{U-Mentalism as a Method of (Typical) Digital Images Non-Standard Positional Numeration base with Isomorphic Many-Bijective Modules Recursive Powers}

Considering the general analogy between the "byte" and the "pixel", with $2^{8}(0-255)$ (256 tonal/chromatic values per each byte in the pixel with 3 pixels), it is known that a single RGB pixel holds, according to the formula $2\left({ }^{8 \times 3}\right)$, i.e., 16.777 .216 tonal values or colors, which is basically the same number of bytes overall combination. This value corresponds to $2^{24}$ in prime factorization, which is a measure very appurtenant due to the imperative of cryptography in the system. The provisory value of the pixel $\left(2^{24}\right.$ color/bytes combinations $)$ is now our minimal symbolic unit, in correlation with the standard binary code, also an (observable noematic) wavelength impression. Now, the density of the pixel equation in agreement with the number of total pixels provides the image resolution in PPI $=\frac{d_{p}}{d_{i}}$ [diagonal resolution in pixels $=\left(d_{p}\right)$; diagonal size in inches $\left.\left.=\left(\mathrm{d}_{\mathrm{i}}\right]\right)\right]$ after the diagonal pixel resolution found through the use of the Pythagorean theorem:

$$
d_{p}=\sqrt{w_{p}^{2}+h_{p}^{2}}
$$

[diagonal resolution in pixels $=\left(\mathrm{d}_{\mathrm{p}}\right)$; width resolution in pixels $=\left(\mathrm{w}_{\mathrm{p}}\right)$; height resolution in pixels $=\left(h_{p}\right)$. Needless to say, we are envisaging any possible variations of measures in the overall structural and functional method.

Also relevant, both physically and symbolically, is the fact that the system in UTM(s) has invariance by synchronicity in all positively-defined and non-accelerating frames of reference (herein "Frames Per Second"= FPS) of the "(typical) digital image" in the system, and likewise the speed of light in the vacuum is the invariant non plus ultra limit of the technology. Therefore, under one such assertion, an 8K Ultra Full HD $(7680 * 4320)$ has 33.177 .600 pixels disposed (in a $16: 9$ ratio, i.e., $2^{4}: 3^{2}$ in prime factorization). In other terms, this means that each $8 \mathrm{~K}$ RGB digital color (FPS) image has 796.262 .400 bits, or 99.532 .800 bytes.

As we are referring to an RGB/binary synthesis within an isomorphic and bijective model, we are most surely asserting a presumed less-to-the-furthest well-ordering recollection of typical digital images, arriving either by general image sensing or general image processing to the UTM(s), from a pool of very different kinds of typical digital images most generally found on the Internet: photos, URL(s) and Web pages, all Turing-machine Frames (FPS) including e-mails and instant messaging, kernel and system logs, OS environment FPS "films", digital TV FPS "films", and every other sort of Turing-machines graphical interface FPS like outdoors and consoles, ATM(s) and GPS(s), CCTV, camera drones, mobiles and tablets, ubiquitous computing things, etc.

Before anything else, the well-ordering of the RGB/binary graphical/digital coeval isomorphic code should be preliminarily understood. Accordingly, below is shown a table of partial wellordering $(16 ; 0-15)$ in a positional numeration base, with inherent many-bijective modules recursive powers. The table is the correspondent to $\frac{256}{16}$ bytes or colors, i.e., $\frac{1}{16}$ of the whole symbolic power of one pixel only under the standard description of the $8 \mathrm{~K}$ model which holds 33.177.600 pixels. It is to be noticed in the table below that the symbolic manipulation under this pixelized part is, hence, only affecting the Blue Byte (in truth, rounded off to even numbers, roughly only $\frac{7680 * 3}{16}$ i.e., $\frac{1}{1440}$ bytes parts of the total in one width or horizontal pixelized line with 7680 pixels, in turn intersected with 4320 pixels in height or vertical lines, in a 16:9 ratio, 
which sums up a total for each FPS, or "(typical) digital image", of $\approx 33.000 .000$ pixels and, therefore, of $\approx 99.000 .000$ bytes.

This is so due to the patterns of the RGB code industry convention, wherein WHITE is $255 * 65536+255 * 256+255=$ \#FFFFFF, and so, by order, RED is $255 * 65536+0 * 256+0=$ \#FF0000, GREEN is $0 * 65536+255 * 256+0=\# 00 \mathrm{FF} 00$, and BLUE is $0 * 65536+0 * 256+255=$ \#0000FF. Coherently, we are generally pointing out to the IEC 61966-2-1:1999 sRGB (standard Red Green Blue) color space associated (and all complementary norms, such as ITU-R BT-601 and ITU-R BT.709 for HDTV), which is, by itself, congruent and abridges other color models, such as the inverse and subtractive $\mathrm{CMY}$, and beyond the human-vision perceiving models "Hexcone" HSV and "Double Hexone" HSL, or really any CIELAB color space, along with $\mathrm{Y} \mathrm{C}_{\mathrm{b}}$ $\mathrm{C}_{\mathrm{r}}$ and $\mathrm{Y} \mathrm{C}^{\prime}{ }_{\mathrm{b}} \mathrm{C}_{\mathrm{r}}{ }_{\mathrm{r}}$ defined in terms of one luma component $\left(\mathrm{Y}^{\prime}\right)$, and two chrominance components, subsuming, in order, somehow overlapping color image pipeline $\mathrm{U}$ (blue projection) and $\mathrm{V}$ (red projection), hence Y'UV. However, any other suitable well-ordering complies and falls as predicted and logically accommodable in the technology.

Here we open a short parenthesis, for an assessment of future technologies. Under the exploratory study of optical or photonic (projecting optical quantum systems) computing power, where UMentalism technology and CA ought to be most perfectible, due to the inherent "cell"-like unit packets of spectrum information, replicable of the RGB "cell"-like "pixel" in the state-of-the-art technology, as long as it is made possible for pre-existing electro-optics devices processing and interconnection, optical interconnection networks, and to a greater degree, U-Mentalism optical computing system and architecture, to accurately and locally measure 256 different spectrum wavelengths and frequency per each RGB color, thus measuring a total of $256^{3}=16.777 .216$ possible different colors per "frame", an unprecedent in depth new avenue for electro-optics or all-photonic (projecting optical quantum) super computation, newly diverged from binary data, is unlatched.

More precisely, under the appointed figures, it should be said that the U-Mentalism optical computing system and architecture ought to be minimally implemented once the camera/processing perceiving/observable UTM(s) vertex and focal-point (1-D) solution of luminance - luminous flux per unit (solid, orthogonal and synchronous) angle, and unit source area or luminous intensity per emitting area unit - thus in controlled, never-subjective but photometric automatic brightness, and in articulation with the (2-D) vertices $\mathrm{X}, \mathrm{Y}$ of chromaticity, hence in full (3D) "film", conceivably within a (3-D) X,Y,Z axis in the CIE (RGB) color space, with perdurable constant and perceived/observable luminance in the UTM(s), corresponds, in general terms, to the standard minimum requisite of realizable spectra(illumination and imaging) various combinations, firstly considering electro-optics, thereafter photonics (projecting optical quantum systems) or, at large, U-Mentalism optical computing. In one such case, what arrives is the practical discarding of the binary code, i.e., the conditions ought to be compulsory for the proper computer graphics palette (and gamut) with 256 shades for each RGB color to be, in obvious self-referential manner, a proper color graphics symbolic index.

Likewise, human-perceiving color models, nevertheless conforming a mathematical model wherein colors are represented as tuples of arithmetic representation, settled on neurophysiology imperatives, the binary code too is, due to the very same neurophysiological causes, a humanperceiving based mediation, moreover challenging enough of higher-level programming languages symbolic design. Stressing the comparison, at a deep systems level, it could be said that the trichromacy or three-different channels and cones of human vision, itself conditioned by the stereoscopy two orbits or cavities binocular vision, is better representative of the symbolic 
International Journal of Web \& Semantic Technology (IJWesT) Vol.12, No.1, January 2021

photo- and neuro-receptivity, in articulation with human-machine and machine-machine communication, than it is the ON and OFF discrete states of the binary code all alone. But what is more truthful is, instead, that one model only ought to be prevalent (from RGB/binary to single RGB), even if both considered can, should, and ought to be concurrent until a full systems symbolic design optimization is physically attainable.

However, a binary code table equivalent will always persist for the abstract drawing of semantic equivalences in photonic computing, in both human-machine and machine-machine communication, yet predicted to be, under one such scenario, relinquished, if not for anything else, because of quantum computing emergence.

Anyhow, at this point we are on the fringe of both pure photonic computation and pure quantum computation at symbolic systems level, insofar, if duly noted and enshrouding quantum superposition, measurement, and entanglement, for instance, looking back to broad 8-bit and 16bit computer graphics ages, the minimal 3-bit RGB relative intensity palette, with $2^{1 \times 3}=8$ colors in total, inherently disposes 4 color cube vertices only, which is, again in symbolic terms, remindful of the two orthonormal basis states $\{|0\rangle,|1\rangle\}$ of the quantum computational basis with the physicalist entangled correspondents pairs into a cube "pixel"-like (in a Bell state) model. Besides, the native determining triads $|0\rangle=\left[\begin{array}{l}1 \\ 0\end{array}\right]$ and $|1\rangle=\left[\begin{array}{l}0 \\ 1\end{array}\right]$ in Dirac's notation for general quantum states of two orthonormal basis states, considering the third-state of superposition which diverges from binary logic, are emulated in the three channels Red, Green and Blue of the RGB color model. Also relevant is, still in a pure imagetic provision, the idea that each vertex of the cube herein under appraisal abstractly bounds a vertical and horizontal polarization under measurement, herein represented by the edges of the cube, in a coherence state. This cogitation is also closer to the consideration of the pure state as represented in a Bloch or Riemann sphere, with mutually orthogonal linear algebra dependent state vectors. It could also be extended to affirm, against this background, that each metric/imagetic/recursive/quantized "frame" would have to be, under the U-Mentalism "O" approach, coincidentally and all together one three pictures of quantum mechanics "frame" (meaning with this Schrödinger's, Heisenberg's, and Dirac's dynamic representations of quantum mechanics, depending on if the constant is, respectively, the observable, both the ket state and density matrix, or none and all in interaction), with great consequences in relation to time-dependency. This is not to be misunderstood in the sense that quantum computation could ever, either trough a universal quantum simulator or a quantum Turing-machine (QTM), or as it might be U-Mentalism QUTM(s), decide on the validation of any arrow of time (from cosmological to perceptual) or any underlying physical extra-theoretical principle, but instead and only that such $\mathrm{M}(\mathrm{s})$ would decide under multiple equivalent and generally uncertainty quantum representational dynamic "pictures", in one celllike "frame". Maybe the only exception to this is the proper equivalent simulation of what can be described as the collapse of time (beyond T-symmetry), and indeed of space time except its representation, in the sense that this cogitation is equally equivalent, and thus all the well-ordered arrows of time (from thermodynamic to quantum, from causal to cosmological) including any $\mathrm{UTM}(\mathrm{s})$ or $\mathrm{M}(\mathrm{s})$ computable and recursive decidability, could all be perceptual. Yet, one such perception could never be produced, as shown, by any sort of UTM(s) or M(s) simulation, neither by any QTM(s), nor indeed by any assigned underlying physics determinacy principle, because, at source, perceptual representation will always continue to be subject of indeterminacy, much beyond model-theoretically time-or spacetime-dependency problems.

This is only to show that even under the exploratory study of optical or photonic (projecting optical quantum systems) computing power, U-Mentalism technology "pixel"-like or, beyond, "jot"- like, or even quantum "bits"-like model - "A bit is a bit. It is either 0 or it is 1 , and that is all there is to it."[3] Chris Bernhardt rightly remarked - or, better said, quantized cell-like 
"frame", are definitely accommodable under the general U-Mentalism color, photon, or quanta "cell"-like exponential-prone basis.

Now, refocusing to the table below, we shall remember that the 8K Ultra Full HD $(7680 * 4320)$ with 33.177.600 pixels (disposed in a 16:9 ratio) with 99.532 .800 bytes, the RGB convention pattern well-orders RED, GREEN and BLUE accordingly, but in U-Mentalism what matters is the binary module correspondent (imagetic and electro-optic operational) mirror. As we shall see, even if U-Mentalism executes (typical) digital images in recollection, ought to fundamentally be an emission RGB/binary technology, and not, though necessary, a binary/RGB mirror system. For instance, all the different 256 BLUE combinations do not exactly all concur to all the different final (perceptual) BLUE colours, but they unequivocally provide $256(0-255)$ different binary module combinations per channel, and RED, GREEN \& BLUE in total provide the different $256 \times 256 \times 256=16.777 .216$ possible and decidable effective modules combinations per FPS.

The inference to the best explanation is simply that the balance and potential for escalation of the binary system $(0,1)$ coupled with 796.262 .400 bits, or 99.532 .800 bytes, in the digital image, is fairly inferior compared to the binary system $(0,1)$ coupled with the 16.777.216 RGB color/tonal combination range in the pixel. The pixel is just the most accessible wavelength and color isomorphic synthesis as of this stage of technological development.

Table 1. RGB 24 Bits Color Calculus, RGB Binary, Hexa, and Ordinal RGB modules

\begin{tabular}{|l|l|l|l|}
\hline $\begin{array}{l}\text { RGB 24 Bits Color } \\
\text { Calculus }\end{array}$ & RGB Binary & Hexa & $\begin{array}{l}\text { Ordinal } \\
\text { module and } \\
\text { Decimal }\end{array}$ \\
\hline$(0 * 65536)+(0 * 256)+$ Blue & $(00000000,00000000,00000000)$ & $\# 000000=0$ & $1^{\text {st }}=0$ \\
\hline$(0 * 65536)+(0 * 256)+$ Blue & $(00000000,00000000,00000001)$ & $\# 000001=1$ & $2^{\text {nd, }}=1$ \\
\hline$(0 * 65536)+(0 * 256)+$ Blue & $(00000000,00000000,00000010)$ & $\# 000002=2$ & $3^{\text {rd }}=2$ \\
\hline$(0 * 65536)+(0 * 256)+$ Blue & $(00000000,00000000,00000011)$ & $\# 000003=3$ & $4^{\text {th }}=3$ \\
\hline$(0 * 65536)+(0 * 256)+$ Blue & $(00000000,00000000,00000100)$ & $\# 000004=4$ & $5^{\text {th }}=4$ \\
\hline$(0 * 65536)+(0 * 256)+$ Blue & $(00000000,00000000,00000101)$ & $\# 000005=5$ & $6^{\text {th }}=5$ \\
\hline$(0 * 65536)+(0 * 256)+$ Blue & $(00000000,00000000,00000110)$ & $\# 000006=6$ & $7^{\text {th }}=6$ \\
\hline$(0 * 65536)+(0 * 256)+$ Blue & $(00000000,00000000,00000111)$ & $\# 000007=7$ & $8^{\text {th }}=7$ \\
\hline$(0 * 65536)+(0 * 256)+$ Blue & $(00000000,000000000,00001000)$ & $\# 000008=8$ & $9^{\text {th }}=8$ \\
\hline$(0 * 65536)+(0 * 256)+$ Blue & $(00000000,00000000,00001001)$ & $\# 000009=9$ & $10^{\text {th }}=9$ \\
\hline$(0 * 65536)+(0 * 256)+$ Blue & $(00000000,00000000,00001010)$ & $\# 00000 \mathrm{a}=10$ & $11^{\text {th }}=10$ \\
\hline$(0 * 65536)+(0 * 256)+$ Blue & $(00000000,00000000,00001011)$ & $\# 00000 \mathrm{~b}=11$ & $12^{\text {th }}=11$ \\
\hline$(0 * 65536)+(0 * 256)+$ Blue & $(00000000,00000000,00001100)$ & $\# 00000 \mathrm{c}=12$ & $13^{\text {th }=12}$ \\
\hline$(0 * 65536)+(0 * 256)+$ Blue & $(00000000,00000000,00001101)$ & $\# 00000 \mathrm{~d}=13$ & $14^{\text {th }}=13$ \\
\hline$(0 * 65536)+(0 * 256)+$ Blue & $(00000000,00000000,00001110)$ & $\# 00000 \mathrm{e}=14$ & $15^{\text {th }}=14$ \\
\hline$(0 * 65536)+(0 * 256)+$ Blue & $(00000000,00000000,00001111)$ & $\# 00000 \mathrm{f}=15$ & $16^{\text {th }}=15$ \\
\hline$(0 * 65536)+(0 * 256)+$ Blue & $(00000000,00000000,00010000)$ & $\# 000010=16$ & $17^{\text {th }=16}$ \\
\hline
\end{tabular}

The well-ordering herein disposed constitutes itself a major resemblance feature with the fundamental theorem of arithmetic, but this time in graphic/digital format, and fundamentally with inherent many-bijective recursive powers under super-computation, greatly emancipating the products of the faculty of imagination, with much greater power of synthesis and scope. Because it is not only analytical, but dialectic, or better said dynamic, it is not only arithmetic, but essentially algorithmic. 
International Journal of Web \& Semantic Technology (IJWesT) Vol.12, No.1, January 2021

Indeed, the unique-prime factorization theorem in arithmetic progression, as well as any chosen unique or non-unique progression containing composites (not necessarily through 8 bits modules) is spontaneously an algorithm of the system, moreover with canonical or non-canonical operations and functions, either cognitive (man-machine noematic-representing) or practical (man-machine evaluation-apt and choice-expressing), where from mathematical and philosophical noemas and judgements are predicated in relation with UTM(s).

In what regards the positional system, enough is said if we declare that the binary radix of the system, congruent with any other numeral system, works with the "octets-bytes", or rather "3 octets -3 bytes", of the "pixel" itself as placed (RGB bytes and color) value notations, with width and height, and ahead time-valued and inter placed-FPS combined index positions, constituting any possible number or algorithm. Let us notice that by programming itself, nonautonomously but spontaneously, a non-standard positional numeral system, in synthesis (presumably also under the unity of apperception in man-machine communication) and synchronicity with a place and time combined valued notations, is present, even if the system is a standard positional numeral system.

At this point, with the value at hand of 99.532 .800 bytes in an 8K Ultra HD digital image, this value corresponds to the total isomorphic RGB digital image-to-binary code ready to be processed by image sensors (impression), and also ahead processors (emission), being in this way clear that the system has only to differentiate $2^{8}$ per byte/per pixel, or $2^{8^{* 3}}$ per pixel locally, instead of the much harsher demand of the linear-dependent CPU, or non-linear CPU-dependent GPGPU general amount of 99.532 .800 bytes per FPS, in one "(typical) digital image".

The next step is, thus, the assessment of the chosen value of 60 FPS (Frames per Second) in conjunction with the present invention, a pretty conservative value to take into account, specially if we consider that the INRS research team has, with the T-CUP, over passed the threshold of 10 trillion FPS, invading the femtosecond scale, i.e., $\frac{1}{1 * 10^{-15}}$ of one second (or quadrillionth of a second). Now, $60(2 * 3 * 5$ in prime factorization) FPS, as soon as it meets the second FPS or frame, is defined as a "movement-image" or "film", pointing out to a value of

$$
60(\mathrm{FPS}) * 60\left({ }^{\prime \prime}\right) * 60\left(^{\prime}\right) * 99.532 .800 \text { bytes }
$$

21.499.085.000.000 $=2.1499085 \mathrm{e}+13$ bytes per hour in one film only in the technology herein presented. The correspondent and conveniently converted value of bytes per hour in one film only is, hence, $2.1499085 \mathrm{e}+25$ Terabytes, or $21.499085 * 10^{15}$ Zettabyes.

It is pertinent to contend that this value is a dense discrete metric measure and, although it can be put forward in synthesis in one film only, in programming algorithmic technical terms it might eventually have been formed by the concurrence of many permuted and/or combined, rather than composed, "films", or as it might be FPS tunnels of "pixels" as wavelength impressions/emissions all throughout every bit and at the full length of the movement-image per hour in one film only under the technology.

Confronting anew with Alan Turing's On Computable Numbers, with an Application to the Entscheidungsproblem, definition - "The machine is supplied with a 'tape' (the analogue of paper) running through it, and divided into sections (called 'squares') each capable of bearing a "symbol" "[2] - the shift to U-Mentalism in the "C" approach is easy to follow if we declare that the "tape" is now film, "squares" are now (FPS) frames, and the "symbol" the graphical/digital movement-image encompassing the necessary RGB/binary " $r$-th bearing of the symbol" in network distributed in as many as possible partial computing UTM(s). 
Attention should also be called to the fact that IDC and Seagate forecast that the global data sphere, which was of 33 Zettabytes in 2018 , will grow to 175 Zettabytes ( $175 * 10^{21}$ bytes)by the coming year of 2025 [4]. In other words, the figure found of $21.499085^{*} 10^{15}$ Zettabyes for U-Mentalism one hour of one film only of processing power return from data is, on its own, $1.2285191 \mathrm{e}+14$ times more than the expected global data for 2025. In point of fact, it would have to elapse 120 years, with each year equally with 2025's 175 Zettabytes + a $100 \%$ growth rate for each year, so that the datasphere would approximate the return result of processing power of the technology for one only film of one hour only thereof encapsulated. The growth rate of data is hereof paramount, as considering if not, figures are that 17.500 years, each with equal 175 Zettabytes of data, would have to pass by to meet one hour only of U-Mentalism, with very conservative parameters for the processing power. In view of this, and on the other way, assuming the quadratic nature of quantum computing worst-case complexity in confront with classical computation, if we envisage the system's complexity complemented with quantum computing complexity, and most specially, counting with the accelerator factor of U-Mentalism on the production of data, the inception in years might be dramatically shorter. What is more, supposing that the technology and CA would work on the full 175 Zettabytes of global data for the year 2025, the super-computation involved would assume 48.611.111 Terabytes per second $\left(4.8611111 * 10^{19}\right.$ bytes per second), which sums up $2^{65.39792}$ per second, already above the capacity of a 64 bits architecture. In truth, in one such case, the technology is imminently conjectured to improve above 1 Exbibyte $(\mathrm{EiB})=2^{60}$ or $1024^{6}$.

Because the S.D. of the technology can be represented by a set forming the symmetric group of the set, which is a bijection from S.D. to itself, and for which every placed octet-byte (binary) element occurs exactly once as the corresponding placed (RGB) image value, S. D.n is the symmetric group under permutation, a broad relation and it is also a function composition under group theory. For the reason of implementation of less-to-the-furthest well-ordered recollection (large numbers arithmetic) and further forward well-ordered collection (at large algorithmic) of typical digital images, onward to be run by as many as possible UTM(s) in a network of UTM(s) on the Internet, a calculus of permutations is needed and, complementarily, it is imperative to calculate a fair assessment of U-Mentalism computational time complexity. With this procedure we are already asseverating the inclusion of crucial demands of the system, such as the use of AI \& cryptography general image processing of typical digital images, apart from general image sensing of typical digital images, as well as the run time complexity in relation with the data memory involved.

\subsection{U-Mentalism (Typical) Digital Images Permutations in Partial and Distributed UTM(S) in a Network, on the Internet}

Once the $8 \mathrm{~K}$ resolution Ultra Full HD (typical) digital image (FPS) bears $(7680 * 4320)$ pixels, which sums up 33.177.600 pixels (width * height), what follows is the application of the formula of permutation having in mind the measurement in pixels

$$
P(n, r) \frac{n !}{(n-r) !}
$$

Thus, $\mathrm{n}^{\text {? }}$ is, really, the number of colors per pixel, which is $256^{3}$ and the correspondent to the combination range, which equals 16.777 .216 color combinations per pixel, while $r^{?}$ is, really, the previous value of $8 \mathrm{~K}$ Ultra Full HD (width * height), i.e., 33.177 .600 pixels. It is very easy to appreciate that the break-up of the two orders of factorials points out to unmanageable numbers, directing both to countable infinite numbers, andO(n!)non-assessing time complexity, regardless of the CA, recursion power or machine. One such calculus is, nevertheless, judiciously desirable 
International Journal of Web \& Semantic Technology (IJWesT) Vol.12, No.1, January 2021

by cause of the intrinsically $\mathrm{O}(\mathrm{n}$ !) factorial time complexity exposition of the system, dragging $\mathrm{O}\left(2^{\mathrm{n}}\right)$ exponential time, and $\mathrm{O}\left(\mathrm{n}^{\wedge} 2\right)$ quadratic time hardness lines, as well as, in middle-way and by order, $\mathrm{O}(\mathrm{n})$ linear and $\mathrm{O}(\log \mathrm{n}) \log$ arithmic times- herein $\mathrm{O}(\mathrm{n}$ !) grows faster as it abridges a constant exponential base 2 -, but on the side of U-Mentalism overlapping solvability, not of classical computation. Simply, the algorithms of U-Mentalism are yet unknown, and the infimum complexity that solves a class of problems is of the same complexity as that of the problem. One such assessment comes even beforehand newly fine-grained analysis, defining the possible class of problems as the set of computational problems of related resource-based complexity, given that time, processing, memory, and more so the relation between them is radically different in UMentalism, however included in Turing-machines computability Application to the Entscheidungsproblem [2].

On the edge, by nature of the intrinsic arithmetic system in U-Mentalism, we could even consider each frame a large number image, reducing the composites factor in $8 \mathrm{~K}$ of 33.177 .600 pixels to one FPS and, consequently, to each one (FPS) large number linear arithmetic progression image, which and in turn transforms the calculus of the combination of colors per pixel per FPS, to one onlylarge number arithmetic mirror per FPS. One such FPS large number image progression would meet a factorial (FPS) table itself of $\mathrm{O}(\mathrm{n})$ linear time complexity, that is made exactly a " $\mathrm{C}$ " imago mundi for the "movement-image" recursion in the system. It is true that the immediate next polynomial running times (quadratic, cubic, $\mathrm{n}^{\mathrm{c}}$, etc) hold important classes of algorithms to discern an unequivocal well-ordered (FPS) large numbers arithmetic progression mirrored (RGB) images, in recollection (composition) and collection (permutations/combinations) of typical digital images. However, U-Mentalism is not a system to solve one such linear progression FPS problem, but instead to solve ahead any algorithm class problems newly defined by the system itself, with inherent new space and time complexity powers, insofar one such linear FPS progression is being expanded in the system.

It is important, under this context, to remember that decidability is based on the localist decidability (even working with non-localist quantum computation by chance) of the pixel isomorphic RGB image of every binary octet. Accordingly, the large number arithmetic (RGB) image mirrors of the system have already exceedingly computing power, all throughout a system where data and processing are positively "C" entangled: data capacity returns processing power, and processing power returns data capacity.

All in all, and luckily, steadily paced performance up to constant verifiable factors is all that is needed under the U-Mentalism system, in a deep and low-level performance requirement RGB/binary enhancement only, wherein, unambiguously, arithmetic progression in FPS follows locally each digit power of two in binary, once decidability in terms of a machine $\mathrm{M}$ or $\mathrm{U}$ is said to be a decidable problem if there exists a corresponding $\mathrm{M}$ or $\mathrm{U}$ which halts on every input with either 0 or 1 , thus low-level feeding the FPS arithmetic progression of large number mirror RGB images. This is, besides all, what makes it not constructive at all, and indeed counter-productive, any glimpse whatsoever over a hypothetical solution based on (typical) digital image decreasing measure overall pixel/bytes reconversion. An aforesaid presumptive choice of lesser resolutionsay, maintaining the 16:9 $=\left(4^{2}: 3^{2}\right)$ ratio, $500 * 281.25$ (width * height) in pixels -would naturally decrease the computational power of the technology and, ergo, the overall scalability of the technology in relation to the cybernetic network on the Internet, under which a minor convolution of data and processing power altogether would impend on time complexity solvability.

What happens is exactly the contrary: the datasphere is too tiny when confronted with the power of U-Mentalism, to the point where well-ordered recollection and collection are pivotal not only to operability, but also to the progress of the technology. 
More importantly, in the localist decidability of the pixel isomorphic RGB image of every binary octet resides the fundamental criterion of difference and repetition at which underlies the XOR or Exclusive OR argument at the root of progression of binary numbers or, indeed, mod 2 addition.

If noticed, the progression $(00,01,10,11)$ corresponds to binary addition, after which completion the next two bits on the left are triggered to shift by half-addition, the same is saying, the double of the previous elements of the series of progression.

In other terms, it corresponds to a not equivalence NEQ difference and repetition operation in binary/RGB isomorphic arithmetic progression, wherein the proper R(ed), $\mathrm{G}($ reen) \& $\mathrm{B}$ (lue) are module operations. In this fashion, the whole (FPS) (typical) digital image becomes a truth-table, for the reason that the lines and the columns (width * height) are themselves a sum operation, needing only a carry-out color/bit to the left when the progression $(00,01,10,11)$ ends.

This would equate having in the binary logic and image sensing (and processing) unit, presumably, a two-color/bits XOR and AND adder. Naturally, because of the need of a full adder circuit for the entire (FPS) (typical) digital image, the binary/RGB isomorphic nature of the UTM(s) would rather, again presumably, be prone to use a two-bits/color XOR, AND and OR. Inasmuch as, for instance, in propositional calculus, laying the foundational bedrock of logic since Aristotle - considering the four different types of categorical propositions in the square of opposition, withstanding the syllogism theory - there are three propositions for each place-valued syllogism figure out of possible four. Thereupon, the possible total number of syllogism modes is four times that number, i.e., 256 logically possible distinct types. Because 256 is the same number of module 8 bits per RGB color in the (FPS) (typical) digital image, what this signifies is that, for the sake of the argument and hypothetically, an (FPS) (typical) digital image is a polysyllogism and a calculus ratiocinator $\mathrm{M}$.

However, and fundamentally, in U-Mentalism the CA is inverse and, thereupon, it is not built on binary/RGB, but instead RGB/binary. Without this judgement, it comes not to be transcendental. It shall produce wavelength colors and forms synthesis, just like púøıৎ (nature).

Due to the localist nature of both the pixel and of the whole (FPS) (typical) digital image, the $\mathrm{RGB} /$ binary isomorphic nature processed in the UTM(s) will be prone to use, not quite an equivalent color summands adder, but instead an every n-ary color/bits RGB imagetic relations instant mirror, filter and mixer, always remembering that in between different modules and (FPS) films the exactly same holds true. What this means is that U-Mentalism is, at each UTM processing, constant metric localist, either in a pixel, a module, or the entire FPS, with equal time-dependent "film" computing power on the previous synchronous and orthogonal base for each (typical) digital image, n-ary modules or bytes.

In truth, the RGB/binary relation in the CA is always affected by a special bottleneck related with the communication with classic computation, as far as other much less grievous than the von Neumann bottleneck, which basically corresponds to the arithmetic logic unit binary mirroring of the RGB image codomain in case only of U-Mentalism scanner-to-printer or Eye-to-Brain, but except for machine learning, not in the case of U-Mentalism printer-to-scanner or Brain-to-Eye. If any bottleneck in the system exists that is worth mentioning it is, inevitably, what we choose to designate the unfolding "O" and "C" philosophy of (time) history bottleneck. The reason behind so is that computational means and resources up to this point of the " $\mathrm{C}$ " state-of-the-art do not produce a reasonable amount of data as to test match the system, which is test halting (time) history itself. Before having a chance of escalation from one hour of one only film in the technology to a film of several hours, years, and even synthesis of the image metric distance, in 
International Journal of Web \& Semantic Technology (IJWesT) Vol.12, No.1, January 2021

the "C" movement-image, in light-seconds, (time) history "O" has, simply, to elapse. In contrast, as seen before, ["C"] computational time complexity in U-Mentalism has equally to elapse, although much more tied with bondless mathematical and dynamical limits, precisely on the grounds of the ["O"] constitutive transcendence on ["C"].

It is, ultimately, by virtue of this assertion that it is more appropriate to make mention of a general U-Mentalism "C"-."O" bottleneck, which is, by and on itself, a rectification of the classical von Neumann bottleneck. To alleviate any remaining doubts, it should be elucidated that the system is an inversion of the von Neumann CA, not only because of the RGB graphical primeval symbolic precedence, but also, amongst other aspects, of the inherent entanglement of data and processing power, which drives high latency, on a U-Mentalism turn, from being orderly unavoidable to well-orderly avoidable.

U-Mentalism, in this fashion, especially due to the diagonal method of computability beforehand, and of well-ordering collection also on the basis of the diagonal method of cryptography and permutation/combination, has a nature of transfinite (FPS) typical digital images, denumerable "image-movement" sets, with inherent cardinals and ordinals equipollence. For the moment it suffices to say that the nature of computable and definable numbers, as for the rest composite or prime numbers in U-Mentalism as " 3 octets -3 bytes" modules, typical digital images, or "films" is, intrinsically, a bijection of the well-ordered set of all finite ordinals in the system $\mathrm{w}_{0}$ to cardinality $x_{0}$. Therefore, an algorithm for an well-ordered collection of typical digital images in the system could easily resort to a typical diagonal on the binary basis and exceptionally reductionist.

Inquisitively, one can picture also, in an Imitation Game [5] register, or in a Turing Test flair, a different dialogical test. We shall call it for now both the U-Mentalist "O" inquiry and the " $\mathrm{C}$ " test. On the grounds that any (human or machine) synthesis of electromagnetic wave-like physical and symbolical impressions, susceptible of being, in turn, emitted in any body or technology, are to be, in quantized electromagnetic wave-like impressions in continuous spacetime, indiscernible in nature, the following questions arise:

Under U-Mentalism "O", we inquire if it is possible to be an observer without photons or any observable frame of reference in spacetime.

Under U-Mentalism " $\mathrm{C}$ " and likewise, we shall test if ever the prior impressed and, thus, emitted UTM(s) wave-like synthesis or images, in spite of the foreign face-to-face relation within a body of a presence in front of it, can be made discernible from the frame of reference of the observer. 


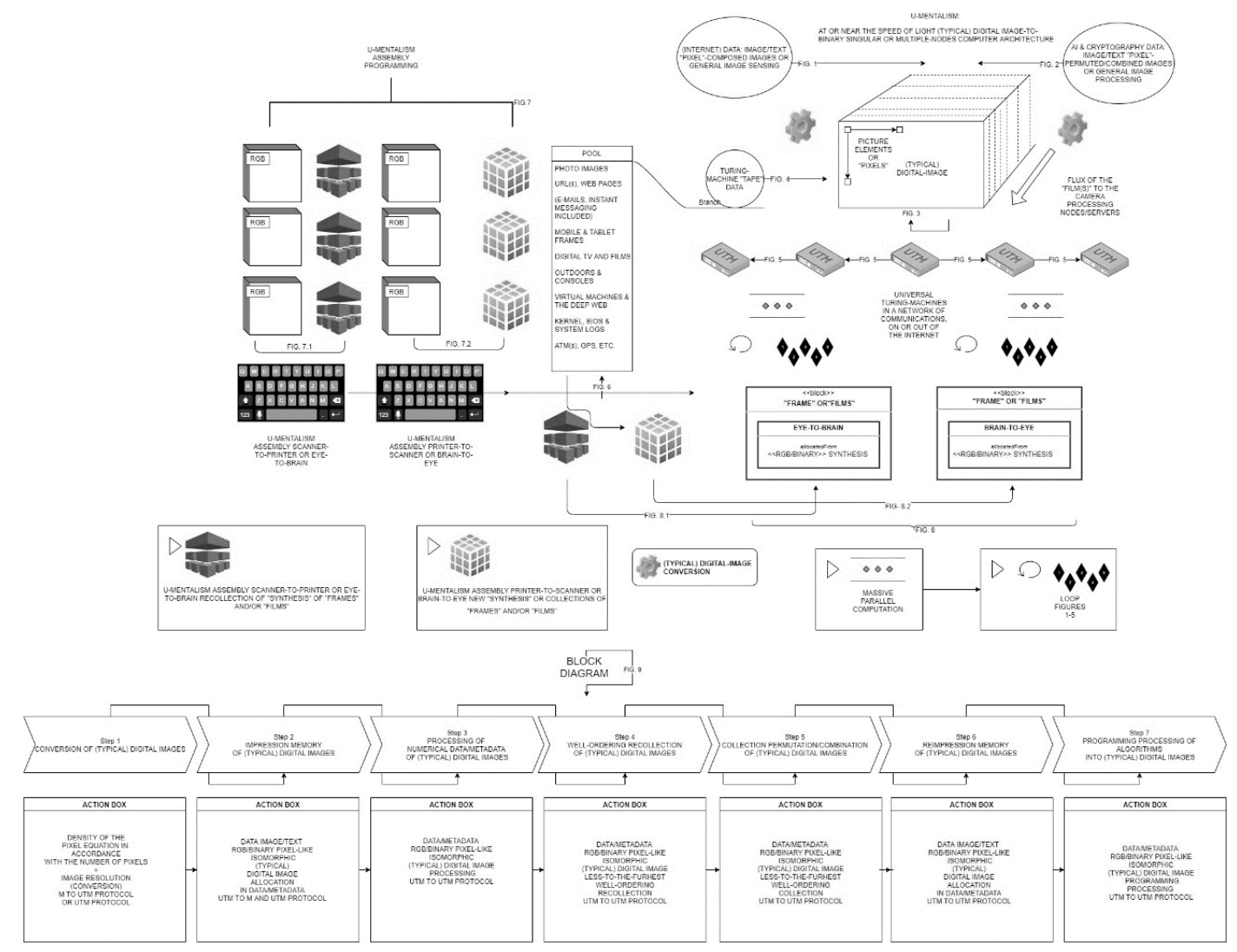

Figure 1. U-Mentalism Computer Architecture Design

\subsection{U-Mentalism UTM(S) Networkanalogy with Current Data $\mathrm{N}^{\circ} 1$ TOP500 Linpack}

Super-computation is often measured or estimated according to the floating-point (FLOPS) (additions and multiplications) computing power in the $\mathrm{M}$, under the numeric and scientific Fortran-derived linear equations in the LINPACK Benchmarks, taken as 64-bit floating-point peak performance. Besides being quite a multi-dimensional problem to address, and essentially a non-distributed supercomputer system ranking, it is the most reliable source for highperformance computing so to have a basis of comparison with the U-Mentalism CA, either distributed or non-distributed, in or out of a network, on or outside of the Internet. This is important to refer, once the CA is developed to be on distributed massive parallel computation with the most possible advanced cryptography methods on each UTM server/node (like opensource blockchain), in a network, on the Internet. Any contrary application of the CA is envisaged as jeopardizing and, indeed, potentially very harming if ill-fated. It shall, hence, be laid open - patere (wherefrom the word patent derives) - for both public inspection and public policies, laws and interests of governments and the people. Once these aspects bring in multivariables of which values is difficult to know, although being much easier to acknowledge approximations, and most specially orientated-guise measures of central tendency, normal distributions probability, and deterministic ranges, our method shall simply attain to the plausible measure for the intensity of the required memory per unit of performance, along the standard of FLOPS and bytes per FLOP (B/F).

Previously, though, in what has to do with the blockchain open-source technology, the idealdesideratus of the technology is to have a (typical) digital modules, "frames" and "films" 
International Journal of Web \& Semantic Technology (IJWesT) Vol.12, No.1, January 2021

(beyond hypertext) U-Mentalism "hypermedia" [6] world wide web service on the Internet itself, wholly constituted as the proper inter-UTM(s) U-Mentalism nodes/servers (miners/theorem solvers/record-keeping services) communication network of networks. In this fashion, UMentalism "C" shall be developed to set off profoundly affecting social, cultural and economic changes, such as social transparency and political trust, law and legal abiding contracts, consensus breaking and activation, counter action to phenomena such as tax evasion and offshores, laundering money and corruption, defense of copyright claims, public registry, thereafter with economic and financial crypto-currencies full securitization.

Blockchain, as follows, shall not to be implemented as an external feat technology, but instead its decentralized, open and distributed ledger with recording of (typical) digital images modules, "frames" and "films" hypermedia ought to account for the UTM(s) imagetic many-bijective isomorphic powers of combinations/permutations in the system, on the Internet, in line with privacy and data protection.

Comprehensively, cryptography in blockchain on the U-Mentalism system, herein understood both as asymmetric-key algorithms and hash functions, and in perspective utter build out of technical knowledge such as testing indistinguishability obfuscation $\mathrm{i} \mathcal{O}$ on the U-Mentalism system, are to beinferred as (typical) digital images RGB-binaryisomorphic modules, "frames" and "films" plain-hypermedia, cipher-hypermedia, hypermedia authentication signatures, and, consistently, imagetic and cinematic hypermedia public and private keys, inasmuch as hypermedia sub-exponential modules in the case of indistinguishability obfuscationi $\mathcal{O}$, in the pursuit of an universal black-box (machine-to-machine communication in the U-Mentalism system).

However all the details and possible conjectures cannot be expanded in this paper, in what relates to the coupling of the system bedrock of U-Mentalism with UTM(s) block(s) explorer services, and all derived "C"-"O" exchanges in a new semantic web design, some philosophical and technical appraisals are likely to be carried out. Along these lines, it is important to express that the pairing of asymptotic $\mathcal{O}$ in U-Mentalism on the Internet, meaning the order as the growth of rate of a function, being commensurable with the basic well-ordering (arithmetic) recollection of (typical) digital images non-standard base with isomorphic many-bijective modules recursive powers, and also the well-ordered (algorithmic) collection of n-modules, -"frames" and -"films", besides the 3D cinematic diagonal method of composition/permutation on a network, all in all in hypermedia design, offer, in general, distinctive cybernetic traits in what regards the relation of cryptography with the system. Sharply, it is preferable to say that the aforementioned is true in relation to encoding, hashing, cryptography and obfuscation, but we choose to address in succinct way to cryptography, upon conclusion that, nonetheless possible different goals are sought after, cryptography alone recapitulates the tripod podium of confidentiality, integrity, and authenticity. Some relevant traits to be pointed out are the following, all of them radically different mainly due to the excessive computing power and processing speed of the technology on one hand, and on the other hand to the all-in-one nature of U-Mentalism on the Internet:

The compactness of the system disallows the typical range of attacks - from the byzantine general problem to the 51 percent attack- and trivializes them, as there is not, in essence, any other possible existing network capable of emulation, and subsequently there is not any rivaling computational power. It is designed never to subside and always evolve insofar photonic and quantum computation are scalable and expandable from the system, with QUTM(s) in UMentalism (not binary, neither linear, but instead "frame"-imagetic and "film"-cinematic), on this wise, being the ground zero for the uttermost radical new shift. Besides, the blockchain technology shall also be made coetaneous with (both arithmetic and algorithmic) well-ordering in 
International Journal of Web \& Semantic Technology (IJWesT) Vol.12, No.1, January 2021

the U-Mentalism system, in one such way that the proof-of-work and broadcasting of previous hashes and nonces, along with the distributed timestamp on a peer-to-peer basis, are compelled to be included in registrable and traceable meta-data on hypermedia basis. The compactness of the system itself is better understood if it is declared that, because an UTM is also a Turing-Machine, or as it might be a QUTM, for that reason UTM(s) on M(s), UTM(s) on UTM(s), or even perhaps QUTM(s) on QUTM(s), are themselves permuted, meaning that computable diagonalization (from computability proper to cryptography) is fully performed on massive parallel supercomputation hypermedia basis. In essence, because of entanglement of data and processing power in the multi-dimensional U-Mentalism system, UTM(s) hypermedia are scalable to proper hyper-physical means, as in the case of QUTM(s), but also DNA molecules in DNA computing, or even Brain-Computer Interfaces. Chiefly, the multi-dimensional composition/permutation, beyond 3D imaging of "films" in the network, allows cryptography methods also to grow exponentially cryptoanalysis hypermedia hardness, on the grounds of multiplied number of imaging and cinematic coordinates, such as under parameter-, configuration- or high-dimensional computationally recognizable spaces.

By virtue of the great excessive computing power in the system, the exponential growth of cryptography methods can be surveyed, and beyond, randomized "film"-modules exponentially augmented, all the more on the basis of hardness decreasing techniques, such as, for example, the use of optical computing aberration methods, or programming methods from computer vision and multiple view geometry algorithms, now in hypermedia super-computation. As far as it is correlated, LED-based advantages are herein indissociable, once energy and color efficiency, onoff cycling and dimming, solid-components resistance and focus are contemplated, if no prejudice comes from color rendition or area light source.

The appointed distinctive all-in-one trait of the U-Mentalism system in a network of networks, on the Internet, is of paramount importance, beyond the orthodox bounds of cryptography and crypto analysis understanding, the reason being that the excessive computing power of the technology can possibly even impend a verging leverage and balance of the classical concepts. As an exemplification, the case in point of obfuscation - "to compile programs into unintelligible ones while preserving functionality" [7] from well-founded assumptions - can, in an illustrative scheme in U-Mentalism, be instead the shifting to deceivingly exponential manifold of unintelligibly malfunctioning programs, as well as epistemic stances in a belief network. Some other relevant ideas are, under the perspective of an entrusting and empowered philosophy of the body, the association of asymmetric cryptography and DNA portrait private-key with cultural, social and political value-exchanges. Again, the RGB/binary isomorphic encoding, matched with the excessive computing power of the technology, opens the door to other conceivable cryptography methods, on top of well-known methods, such as onion routing or secure multiparty computation, most of them being in close relation with the multi-dimensional and cinematic nature of "films", or even cold secret cipher-hypermedia "frames" or "films", but principally to the all-in-one nature of the U-Mentalism technology in the network of networks, i.e., the Internet, which is turned impossible to override.

Therefore, as much as we can be conclusive in relation to the proper informatic cybernetic architecture of U-Mentalism in relation to cryptography and cryptographic techniques, ahead of dealing directly with the chosen super-computation $M(s)$ benchmark suite $n^{\circ} 1$ supercomputer, is that the proper reach of hypermedia and cinematic RGB/binary encoding run by UTM(s) on M(s) on the Internet, drastically alters the medium and range of classical limits, mostly because of the substitution of proofs by targets, with short-lived approximative limits to such computational processing power and system ipseity newly investigated bounds. 
International Journal of Web \& Semantic Technology (IJWesT) Vol.12, No.1, January 2021

Currently, the $\mathrm{n}^{\mathrm{o}} 1$ position of the $55^{\text {th }}$ TOP 500 following the LINPACK benchmarks suite (June $2020)$ is the Fugaku petascale $\left(10^{15}\right.$ floating-point operations per second $=1$ petaFLOPS, i.e., a thousand million millions 64 bits operations per second) supercomputer. The Fugaku holds 415 petaFLOPS with a 158,976 (two types of) nodes Fujitsu TofuD, 6D mesh/torus Interconnect, in a A64FX CPU (48+4 core) per node CA, with a second-generation High Bandwidth Memory (HBM2) of $32 \mathrm{GiB} /$ node.

Our next step is, thus, by multiplying Fugaku's instance of cores * nodes $(52 * 158,976$ $=8.266 .752$ ) find the equivalent processing power of the very same number of $\mathrm{UTM}(\mathrm{s})$ cameras/computer processing nodes/servers and later, having in mind that the Internet has around 50.000.000.000 nodes, well above the $10^{12}$ to $10^{24}$ FLOPS of all the existent computers (2015), at the end reasonably cut the latter figure by reason of factors such as entrance in the industry, price and energy, besides any hindering variables, thus obtaining a fair value for the $\mathrm{CA}$ implementation in the network of networks, i.e., on the Internet.

First off, the value of $21.499085 * 10^{15}$ Zettabytes of one only "film" of one hour in UMentalism divided by the number of cores $*$ nodes $(52 * 158,976=8.266 .752)$, equals $2.6006689 \mathrm{e}+15$ Zettabyes, which converted is $2.6006689 \mathrm{e}+15$ Petabytes. Therefore, assuming a 64 bits operation, we have roughly 325083615.064 PetaFLOPS per each server/node out of 8.266.752 in the technology, if U-Mentalism was to have the same number of nodes/servers on the Internet, presumably settled for a start on an Internet with an even much bigger number of nodes. And even if there would never be the same number of servers as nodes, in reality and at present the Internet detains around 50.000.000.000 nodes.

In abstract, the value divided by the computer performance of the Fugaku supercomputer indicates that one only hour of one only "film" in the technology would equate to 783334.012203 times the $\mathrm{n}^{\circ} 1$ position of the TOP 500 Linpack benchmark as of October 2020. In reverse manner, we could affirm that the 415 overall petaFLOPS of the Fugaku supercomputer, compared with the 597196805556 Zettabytes/per second (or 597196.805556 Petabytes/per second) in U-Mentalism, stipulates that a presumed value in 64 bits (74649.6006944) petaFLOPS/per second in U-Mentalism is, in itself, 179.87855589 times more than the overall performance of the Sugaku supercomputer.

The extreme low latency of the invention is best shown if we divide the overall abstract performance of U-Mentalism of petaFLOPS/per hour $(2.6006689 \mathrm{e}+15)$ by the actual number of Internet nodes (50.000.000.000). The result is 52013.378 petaFLOPS per each one out of 50.000.000.000 Internet nodes, when indeed, chances are not only that the divisor will be much larger, but essentially that the number of typical digital images reaching the system will be exponentially wider. Confronting with the Sugaku supercomputer, this would mean that at each one of these 50.000.000.000 nodes, it would be instantiated 125.333440964 times more the performance peak of the Fugaku supercomputer in PetaFLOPS.

Nevertheless, because the hindering variables are numerous and immense, even though we are experimenting values with greatly sub-optimized inherent values (pixel resolution, FPS, Hz, and subordinate processing-time of the technology to one-hour only), we shall now, thinking ahead the barriers to the entrance in the industry in terms of price, energy, etc., cut the preliminary values to around $20 \%$. Thus, the result at hand is, under one such $20 \%$ cut under the very same parameters, of 65016.7225 PetaFLOPS for each one out of 50.000.000.000 Internet nodes, which equates to 100.266752771 times more the performance peak of the Fugaku supercomputer, measured in PetaFLOPS for the very same value of each one out of the 50.000.000.000 Internet nodes. 
International Journal of Web \& Semantic Technology (IJWesT) Vol.12, No.1, January 2021

One such technology shall be exclusively scientifically-driven. In fact, in terms of the storedprogram concept, we can designate it, in indifferentiable manner, ( $\varphi v ́ \sigma ı)$ ) science, if granted that the overall feedback and cybernetic loop mechanism, that we choose to call an "algorithmitron", i.e., an accelerating mechanism for all classes of algorithms, and thus a procedure on its own, is itself well-ordered within the extensional and intensional self-image of man and the cosmos that is ( $(v 01 \varsigma)$ nature. The extensional and intensional philosophical synthesis and programing regulative idea shall be explored in recurrence and recursively.

Very concretely, the actual example provided by the current use of the $\mathrm{n}^{\circ} 1$ TOP 500 Linpack benchmark Fugaku Japanese supercomputer, which has presently been used for COVID-19 research, and the $\mathrm{n}^{\circ} 2$ TOP 500 North-American supercomputer Summit, whose current work with scientific impact is on various levels (deep learning for human systems biology, plasma fusion simulation, combustion in turbulent environments, stellar astrophysics nuclear burning, cancer treatment and surveillance planning, high-temperature superconductors) are nothing but just a pale coup d'oeil of what can be, at a greater extent, achieved with the forthcoming fabrication. By all means, U-Mentalism participative and all-engaging cultural-scientific accelerator, social and technological, financial and political, inter-dependability and transparency, shall act as new measures for the human. We have to remember, for that purpose, that a world with a 24 hours "film" in the technology, however $1.2285191 \mathrm{e}+14$ times the value of 175 Zettabytes (estimation of global data for 2025) with the necessary equivalent data input, thus dependent on the U-Mentalism "C"-"O" bottleneck, would grant the CA with a processing power of $5.1597804 \mathrm{e}+17$ Zettabyes $\left(21.499085 * 24 * 10^{15}\right)$. The same is valid for "films" of several years, and even of the "image"-distance in light-seconds, most assuredly in prospective proper physicalist-reductionist underpinnings, rather than merely technological.

Yet, more frequently than not in dialectic terms, time and history itself naturally supersede and are transcendent in relation to any object (such as technology). In fact, the author suggests that the contemporary crisis in philosophy of science ( $\left(v \sigma^{\sigma} \varsigma\right)$ points out to the extreme of that reality. If any peak in civilization was possible to be found, it definitely was the period from the birth of Classical Greece up to the end of the Hellenistic period, from classical Athens to the Hellenistic Alexandria. It worked as a natural philosophy ( $(v 01 \varsigma)$ explosion or radiation, all throughout two millennia with tergiversated and fragile-weaved, often sinuous paths on the edge of eradication, as epiphenomenon's echoes throughout the Roman and Byzantine, Islamic and Indian Empires, before the turning from Medieval times to the European Renaissance, and thereupon Modernity and Contemporaneity, that was afterwards, arguably, to see its dimmest resonate and last hour in $\mathrm{XX}^{\text {th }}$ century Vienna. This serves to explain the non-mutual relation between progress and technology. More so, sometimes the value of a technology is best evaluated if tested against the worst demeanors and actions known to the history of civilizations. For example, in substitution of a colossal computing and processing power, the technology of U-Mentalism could benefit more from improvements in cryptography, or primarily human-agent decisions.

Coming to think of it, and bearing in mind imaging and sensing technology latent in UMentalism - metal-oxide-semiconductor (MOS) based charged-coupled devices (CCD), and active-pixel sensors (CMOS) in the present state-of-the-art, prior to the reductionist more general "cell"-like synthesis-we can think of two unexpected, but conceivable and tenable breakthroughs cognate with the technology that are worth to be referred.

One is, definitely, the use of biopolymers as paper for the use of electronic applications, namely paper transistors recurring to metal oxide semiconductor (MOS), complementary (CMOS) circuits, and eventually transparent conductive oxides, i.e., paper-based electronics or papertronics [8,9]. Most importantly in the case of a simple and universal device architecture in correlation with the novel U-Mentalism CA, it could literally be possible having always and ever 
International Journal of Web \& Semantic Technology (IJWesT) Vol.12, No.1, January 2021

a paper copy of every book in the world in the same paper organic substrate, also electronic component (dielectric), and charge storage media, an upturn revival of the inceptive idea of the Great Library of Alexandria and Mouseion since Ptolemy Soter I, center of Hellenistic civilization and epitome of Classical Greece, where the study of natural philosophy ( $\varphi v ́ \sigma ı \varsigma)$ found

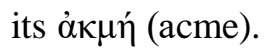

The other conceivable breakthrough is directly correlated with the possible use of transparent oxide electronics as a backbone to U-Mentalism Assembly Language programming. Because in U-Mentalism there will be the need to instruct in symbolic RGB/bytes machine code modules, "frames" and "films" through instances of time in tunnels of "pixels" or, in fact, any other instances of "cell"-like exponential-prone alike basis, having access to novel semi-conductor amorphous oxides or applications with high transparency and electrical conductivity, can open the gates to create an endless array of philosophical and programming short-cuts over typical digital images. In reality, beyond Thin Film Transistors ("active matrix" TFT), Liquid Crystal Display ("passive matrix" LCD), and Organic Light Emitting Diode (OLED), the transparent semiconducting oxides (TSOs) and transparent conducting oxides (TCOs) can help the technology to directly assemble the building block-structure-luminous response mechanism itself, bridging optoelectronics with programming, and possibly breaching into Photo-Voltaic modules (PV or solar panels) or electronics Organic Solar Cells (OSC), including polymer solar cells.

Lastly, the author would like to reiterate the U-Mentalism "O"-to-"C" cybernetic analogy with photosynthesis, the very definition of "synthesis of light", already expanded in a preliminary paper, in all likelihood with improved understanding as of now close to the conclusion:

U-Mentalism is mainly intended to be a programming synthesis of light through typical (digital) images, organized as symbolic-informational truth-equivalent programming language abstracts. Photosynthesis puts together a synthesis of light, carbon dioxide and water into glucose at reaction centre proteins with chlorophyll (digital images), wherein to the fore roots have absorbed water (computability) from the soil, through the stem (programming language abstracts and paradigms) and through the leaves (programming languages). This is why to the exact chlorophyll complementary light (diagonalization) absorbance centre chloroplast organelle (pixel) there is, at large, a leaf lamina (frame), as a surface area to capture the light, under light's every possible and each necessary time-image. There is, in the overall process of photosynthesis, a light-dependent cycle and a light-independent cycle. In the light-dependent or light cycle (scanner-kinescope), as an effect, short-term stores of energy are produced, enabling their transfer to drive other reactions (computer vision \& multiple-view geometry; U-Mentalism Recollection), while in the light-independent cycle (printer-iconoscope; U-Mentalism Collection), the so called Calvin cycle, the atmospheric carbon dioxide is incorporated into organic carbon compounds (UMentalism Assembly Language Programming), and dependent on the previous light-dependent reactions (semantic isomorphic correspondence), are then used to form further carbohydrates, such as glucose, the most important source of energy metabolism in bioenergetics (cybernetics) [1].

\section{Conclusions}

In the present study, following closely the theoretical and practical keystone of the provisional Patent Application at INPI (Portuguese Institute of Industrial Property) ( $\left.{ }^{\circ} 116408\right)$, designated as U-Mentalism, a method to perform computation at or near the speed of light, resorting to "(typical) digital image" RGB-to-binary in singular or multiple nodes/servers in a network, on the Internet, in its entirety a philosophically-meaningful new computer architecture, is displayed its simplest baseline, adjustable for the research and industry communities. Foremost, the proper discrepancy between the imagetic frame of reference of U-Mentalism in relation to the 
International Journal of Web \& Semantic Technology (IJWesT) Vol.12, No.1, January 2021

"O(ntological)" and " $\mathrm{C}$ (omputational)" approaches is elucidated. No substitute of the latter can prepare ahead the in-depth comprehension of the intrinsic method of typical digital images coincident with non-standard positional numeration base with isomorphic many-bijective modules recursive powers. Ensuing, typical digital images permutations in partial and distributed $\mathrm{UTM}(\mathrm{s})$ in a network, on the Internet, is shown to be the proper context for the technology to be undertaken, which suits the passage to a vaguely prosaic, but matter-of-fact indisputable, comparison of the fundamentally futuristic trait of the invention with the current data $n^{\circ} 1$ TOP500 Linpack supercomputer as of November 2020, the Fugaku supercomputer at the RIKEN Center for Computational Science in Kobe, Japan.

\section{ACKNOWLEDGEMENTS}

This work is funded by national funds through FCT - Fundação para a Ciência e a Tecnologia, I.P., in the frame of the project FCT/UIDB/00678/2020

\section{REFERENCES}

[1] Homem, Luís, (2019) “What is U-Mentalism?”, Journal of Advances in Computer Networks, Vol. 7, No. 1, pp.18-24.

[2] Turing, Alan M., (1937) "On Computable Numbers, with an application to the Entscheidungsproblem", Proceedings of the London Mathematical Society, 2, 42 (1), pp. 230-65.

[3] Bernhardt, Chris, (2019) Quantum Computing for Everyone, The MIT Press.

[4] Reinsel, David \& Gantz, John \& Rydning, John, Data Age 2025," The Digitalization of the World, From Edge to Core", An IDC White Paper - \#US44413318, Sponsored by Seagate pp. 1-24.

[5] Turing, Alan M., (1950) “Computing Machinery and Intelligence”, Mind, LIX (236) pp. 433-460.

[6] Tim Berners-Lee (2000) Weaving the Web, The Original Design and Ultimate Destiny of the World Wide Web, HarperCollins.

[7] Aayush Jain \& Huija Lin \& Amit Sahait (2020) "Indistinguishability Obfuscation from WellFounded Assumptions", UCLA Center for Encrypted Functionalities, and NTT Research.

[8] Barquinha, Pedro \& Martins, Rodrigo \& Pereira, Luis \& Fortunato, Elvira, (2012) "Transparent Oxide Electronics, from Materials to Devices" Wiley, a John Wiley \& Sons, Ltd., Publication

[9] Martins, Rodrigo \& Gaspar, Diana \& Mendes, Manuel J. \& Pereira, Luis \& Martins, Jorge \& Bahubalindruni, Pydi \& Barquinha, Pedro \& Fortunato, Elvira (2018), "Papertronics: Multigate paper transístor for multifunction applications", Applied Materials Today 12 (2018) pp.402-414

\section{AUTHOR}

Luís Homem (Lisbon, 21/12/78) has a degree in philosophy (2005) and a Ma degree in natural and environmental philosophy (2008) at the University of Lisbon, having also completed a Ma degree in logic and philosophy of science at the University of Salamanca with the thesis "Topics in Programming Languages, a Philosophical Analysis through the case of Prolog" (2012). He has also a PhD in logic and philosophy of science at the University of Salamanca with the same title thesis "Topics in Programming Languages, a Philosophical Analysis through the case of Prolog" (2018).

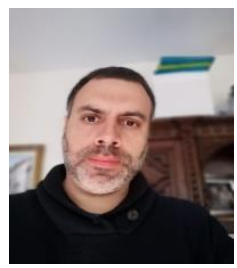
Being a doctorate integrated member of the Center for Philosophy of Sciences of the University of Lisbon (CFCUL), the author has developed research mainly in philosophy of logic, science and language. 